\title{
Supercompensation in Elite Water Polo: Heart Rate Variability and Perceived Recovery
}

\section{() (i) $(\mathrm{\theta})$}

Authors

Petros G. Botonis ${ }^{1}$, Ilias Smilios ${ }^{2}$, Argyris G. Toubekis ${ }^{3}$

\section{Affiliations}

1 Department of Aquatic Sports, School of Physical Education and Sports Science, University of Athens, Greece, Athens, Greece

2 Physical Education and Sport Science, Democritus University of Thrace, Komotini, Greece

3 Physical Education and Sport Science, University of Athens, Athens, Greece

\section{Key words}

autonomic nervous system, monitoring, fatigue, teamsports players

$$
\begin{array}{ll}
\text { received } & 24.02 .2021 \\
\text { received } & 26.04 .2021 \\
\text { accepted } & 28.04 .2021
\end{array}
$$

\section{Bibliography}

Sports Medicine International Open 2021; 5: E53-E58

DOI 10.1055/a-1494-9254

ISSN 2367-1890

2021. The Author(s).

This is an open access article published by Thieme under the terms of the Creative Commons Attribution-NonDerivative-NonCommercial-License, permitting copying and reproduction so long as the original work is given appropriate credit. Contents may not be used for commercial purposes, or adapted, remixed, transformed or built upon. (https://creativecommons. org/licenses/by-nc-nd/4.0/)

Georg Thieme Verlag KG, Rüdigerstraße 14,

70469 Stuttgart, Germany

\section{Correspondence}

Petros G. Botonis

Department of Aquatic Sports

School of Physical Education and Sports Science

University of Athens

Greece

41, Ethinikis Antistasis Ave

17237 Dafni, Athens

Greece

Tel.: + 302107276065, Fax: + 302107276065

pboton@phed.uoa.gr

\begin{abstract}
We examined the association of heart rate variability assessed with the logarithm of the root mean square of successive differences (LnRMSSD) and perceived recovery status of nine elite water polo players with the fluctuations of the internal training load (ITL). ITL, post-wakening LnRMSSD, and measures of perceived recovery were obtained across one regeneration week, during two mesocycles of intensified preseason training (PR1, PR2) and during two mesocycles of in-season training (IN1, IN2). ITL at PR1 and PR2 was increased by $60-70 \%$ compared to regeneration week $(p<0.01)$ and was reduced by $30 \%$ at IN1 and IN2 compared to PR1 and PR2 ( $p<0.01)$. Weekly averaged

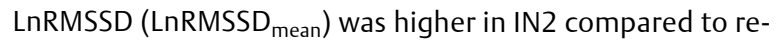
generation week and PR2 ( $<<0.01$ and $p<0.05$, respectively). Perceived recovery was higher at IN1 and IN2 compared to PR2 $(p=0.01$ and $p<0.001$, respectively). ITL correlated with LnRMSSD in the preseason $(r=-0.26, p=0.03)$. Nonetheless, similar association was not apparent during the in-season pe$\operatorname{riod}(r=0.02, p=0.88)$. Cardiac autonomic perturbations may not occur when an increment of internal training load is less than $60-70 \%$. However, the reduction of training load in season by $30 \%$ improves both LnRMSSD $_{\text {mean }}$ and perceived recovery status, implying that training periodization may lead players in supercompensation.
\end{abstract}




\section{Introduction}

In water polo, preseason training encompasses various types of training that aim to improve players' physical conditioning, and technical and tactical efficiency [1]. Following preseason training, a period of reduced training load is usually applied aiming to reduce fatigue and facilitate players' readiness to play. However, in an elite teamsport setting the preseason period is typically brief and players are exposed to abrupt increases in training load [2], making them susceptible to overreaching. In addition, a demanding training and competition schedule during the in-season period may hinder players' recovery and increase fatigue accumulation. Therefore, training monitoring using accurate and easy-to-use tools is indispensable for tracking and managing players' fatigue and recovery status.

Vagally mediated heart rate variability (HRV) is considered a global index of homeostasis mirroring cardiovascular recovery after a training session [3]. The logarithm of the root mean square difference particularly of successive RR intervals (LnRMSSD) reflects cardiac parasympathetic modulation and is considered a fatigue-sensitive marker [3]. Accordingly, LnRMSSD has been used in various team sports for tracking individual and group adaptations to training [4-8]. In water polo particularly, Botonis et al. [4] demonstrated that daily measures of LnRMSSD were useful in tracking cardiac autonomic disturbances and changes in the recovery status of international-level water polo players. In soccer also, weekly averages (LnRMSSD mean) and the coefficient of variation (CV) of LnRMSSD ( LnRMSSD $_{\mathrm{cv}}$ ) have been used to measure players' training response [8, 9].

High training loads coincide with decreases in LnRMSSD $_{\text {mean }}$, increases in LnRMSSD $_{\mathrm{cv}}$, and concomitantly with increases in players' perceived fatigue $[9,10]$, yet this is not a generally accepted view [8]. In water polo, however, a recent short-term observation [4] indicated that the fluctuation of LnRMSSD is not associated with the respective fluctuation of the training and match load, implying that there is no dose-response relationship between LnRMSSD and training load. Thus, a long-term surveillance of training and competition load is warranted to examine the relationship between training load and HRV-related parameters in elite water polo players.

Various studies have examined the usefulness of HRV and subjective measures in identifying training responses and players' fatigue/recovery status, but relatively limited studies exist on elite team-sports players $[11,12]$. In addition, the majority of previous studies include short-term ( $<5$ weeks) or non-systematic (only prepost measurements were employed) observations. The convenience of longitudinal (> 8 weeks) and systematic monitoring of training using HRV-related measures combined with subjective responses in elite team-sports, and in water polo particularly, remains to be elucidated. Therefore, the purpose of the present study was to examine if $L_{n R M S S D}$ mean, $L n R M S S D_{C V}$, and perceived recovery status of elite water-polo players are associated with the fluctuations of the internal training load assessed with the session-RPE method during a preseason and in-season training period.

\section{Materials and Methods}

\section{Participants}

Nine elite male water-polo players (age: $25.6 \pm 4.7$ years, body mass: $94.4 \pm 8.2 \mathrm{~kg}$, stature: $191.9 \pm 12.5 \mathrm{~cm}$ ) took part in the study.
All players had long training experience ( $>7$ years of systematic water polo training) and participated in national league matches and in the LEN European Champions League tournament. The collection data period started at the end of August 2019 and was terminated at the end of October 2019. All participants provided written informed consent prior to the commencement of the study. The experimental protocol was approved by the faculty review board (1107/13-03-2019) and conformed to the Declaration of Helsinki. Procedures were performed in accordance with the ethical standards of the International Journal of Sports Medicine [13].

\section{Experimental design}

The experimental design of the study is depicted on $>$ Fig. 1 . The players were monitored throughout a preseason period ( 5 weeks) as well as at the initial stages of the in-season period (4 weeks). The first week of the preseason period was considered as a regeneration week (from August $26^{\text {th }}$ to September $1^{\text {st }}$ ). Thereafter, the training consisted of 2 mesocycles of preseason training (PR1 from September $2^{\text {nd }}$ to September $15^{\text {th }}$, PR2 from September $16^{\text {th }}$ to September $29^{\text {th }}$ ), which were followed by the in-season training period consisting of 2 mesocycles of reduced training load (IN1 September $30^{\text {th }}$ to October $15^{\text {th }}$, IN2 October $16^{\text {th }}$ to 31 October $\left.31^{\text {st }}\right)$. A summary of the training implemented during the preseason and in-season is presented in > Table 1. Resting HRV was measured in the training facility three times a week (except for the regeneration week, wherein HRV was measured four times). During the monitoring period, participants rated their perceived recovery status each morning upon awakening [14]. Perceived recovery was assessed on a scale of 0 (very poorly recovered/extremely tired) to ten (very well recovered/highly energetic) with players asked "how do you feel?".

Internal training load (ITL) was measured 30 -min post-training by multiplying the rating of perceived exertion (RPE) with training session duration (session RPE) [15]. This method of training load quantification has been previously validated in elite youth waterpolo players [16]. Moreover, the ITL of each match was calculated by multiplying session RPE provided by each player 30 min postmatch with the match duration played by each one. Throughout the distinct training phases, the perceived recovery status [17] was obtained from each player every morning upon awakening (at approximately 08:00 a.m.).

Heart rate (HR) was measured thirty minutes after waking and before the beginning of the morning training session (08:45 a.m.) in a temperate and quiet room with participants remaining seated, silent, and relaxed. Participants were instructed not to consume food or coffee prior to the initiation of the measurements. After allowing $1 \mathrm{~min}$ for stabilization (stabilization period was excluded from the analysis), the RR intervals were measured for three consecutive minutes. RR interval data were recorded using a Polar V800 (Polar, Kempele, Finland) with a Polar H7 chest strap. RR recordings were downloaded via accompanying Polar software (Polar Flow; Polar) and later were analyzed for the LnRMSSD with an HRV analysis software (Kubios HRV Standard 3.3.0 Software; Biomedical Signals Analysis Group, University of Kuopio, Kuopio, Finland). Occasional artefact noise was automatically replaced with the interpolated adjacent RR interval values (very strong filter power). Ln$\mathrm{RMSSD}_{\mathrm{cv}}$ was calculated intra-individually as the standard deviation 
- Table 1 Characteristics of training applied in different phases of preseason and in-season periods.

\begin{tabular}{|l|l|l|l|l|l|l|l|l|l|}
\hline & $\begin{array}{l}\text { Training } \\
\text { volume (min) }\end{array}$ & $\begin{array}{l}\text { Training sessions } \\
\text { (number) }\end{array}$ & $\begin{array}{l}\text { Triple } \\
\text { session days }\end{array}$ & $\begin{array}{l}\text { Double } \\
\text { session days }\end{array}$ & $\begin{array}{l}\text { One } \\
\text { session day }\end{array}$ & $\begin{array}{l}\text { Number of } \\
\text { matches }\end{array}$ & $\begin{array}{l}\text { Rest } \\
\text { days }\end{array}$ & $\begin{array}{l}\text { Total session } \\
\text { RPE (mean } \pm \text { SD) }\end{array}$ & $\begin{array}{l}\text { Mean session } \\
\text { RPE (mean } \pm \text { SD) }\end{array}$ \\
\hline RW & $765 \pm 0$ & 10 & 2 & 1 & 2 & 0 & 2 & $3635 \pm 251^{*}$ & $519 \pm 36^{*}$ \\
\hline PR1 & $2100 \pm 0$ & 25 & 4 & 6 & 1 & 0 & 3 & $12203 \pm 730 \#$ & $872 \pm 52 \#$ \\
\hline PR2 & $1970 \pm 4$ & 24 & 4 & 5 & 2 & 4 & 1 & $11536 \pm 497 \dagger$ & $824 \pm 35 \dagger$ \\
\hline IN1 & $1671 \pm 8$ & 20 & 3 & 3 & 5 & 3 & 2 & $9176 \pm 648$ & $573 \pm 41$ \\
\hline IN2 & $1778 \pm 10$ & 21 & 3 & 4 & 4 & 4 & 1 & $9497 \pm 781$ & $594 \pm 49$ \\
\hline
\end{tabular}

RW, regeneration week; PR1, first mesocycle of pre-season training; PR2, second mesocycle of pre-season training; IN1, first mesocycle of in-season training; IN2, second mesocycle of in-season training; * significantly lower compared to the other training phases, $p<0.001$; \# significantly higher compared to the other training phases, $\mathrm{p}<0.01$, $\dagger$ significantly higher than RW, IN1, and IN2, $\mathrm{p}<0.001$; session-RPE, calculated internal training load.

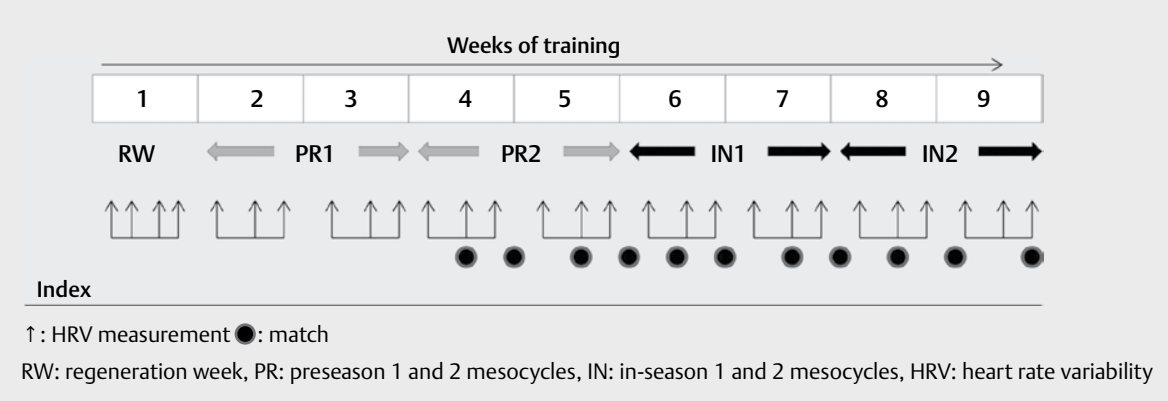

- Fig. 1 Schematic presentation of the experimental protocol.

of the LnRMSSD values obtained in each training phase divided by the mean and multiplied by 100 .

\section{Statistical analyses}

All results are expressed as mean \pm standard deviation. Variation in LnRMSSD, perceived recovery, and ITL values across the training phases was evaluated using one-way repeated measures analysis of variance. A Tukey's honest significant difference post-hoc test was employed to detect specific differences between means. As a measure of effect size, Cohen's $d$ was calculated and values of 0.20 , 0.50 , and above 0.80 were considered small, medium, and large, respectively. Correlations between training load and LnRMSSD values across time were analyzed for each player using Pearson's $r$ correlation coefficient and for the total sample using the correlation coefficient for repeated measures [18]. Significance level was set at $\mathrm{p} \leq 0.05$.

\section{Results}

- Table 1 represents the average ITL value for each distinct training phase. In regeneration week, ITL was lower compared to all the other mesocycles ( $p<0.01, d=1.24-8.06)$. Moreover, ITL was higher in PR1 and PR2 compared to both IN1 and IN2 mesocycles $(p<0.01$, $d=9.91-10.82$ ). No differences in ITL were observed between PR1 and PR2 as well as between IN1 and IN2 ( $>0.05)$. Individual values

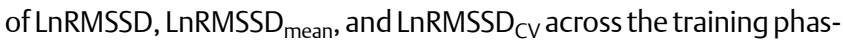
es are displayed in > Table 2 . LnRMSSD $_{\text {mean }}$ was higher in IN2 compared to regeneration week and PR2 $(p<0.01$ and $p<0.05$, respectively; $d=1.66$ and $d=1.33$, respectively). LnRMSSD ${ }_{\mathrm{CV}}$ did not differ among the training phases ( $p=0.19$, $>$ Table 2$)$. A significant main effect was observed for the perceived recovery values $(p<0.001)$, which were higher at IN1 and IN2 compared to PR2 ( $p=0.01$ and $p<0.001, d=1.74$ and $d=1.00$, respectively), and similar perceived recovery values were found among the other training phases ( $p>0.05$, > Table 2).

- Table 3 depicts individual and group correlations between the ITL recorded the preceding one to two days of training with the subsequent LnRMSSD $_{\text {mean }}$ during the preseason and in-season period. Low but significant associations between ITL and LnRMSSD $D_{\text {mean }}$ were found during the preseason $(r=-0.26, p=0.02)$, but a trivial relationship was observed during the in-season period $(r=0.02, p=0.88)$.

\section{Discussion}

The present study examined if $\mathrm{LnRMSSD}_{\text {mean }}$, LnRMSSD $\mathrm{CV}$, and perceived recovery status of elite water-polo players are associated with the fluctuations of the internal training load, assessed with the session-RPE method during a preseason and in-season training period. The principal findings of the study are: i) $L_{\text {nRMSSD }}$ changed substantially across the training phases showing its highest values in the second mesocycle of in-season; conversely, the fluctuation of $\mathrm{LnRMSSD}_{\mathrm{CV}}$ showed no significant changes throughout the training phases; ii) the perceived recovery status of the players did not change during preseason, although it increased during in-season training; and iii) a low relationship between ITL and LnRMSSD was found in the preseason period, whereas a similar association was not apparent in the in-season period. 


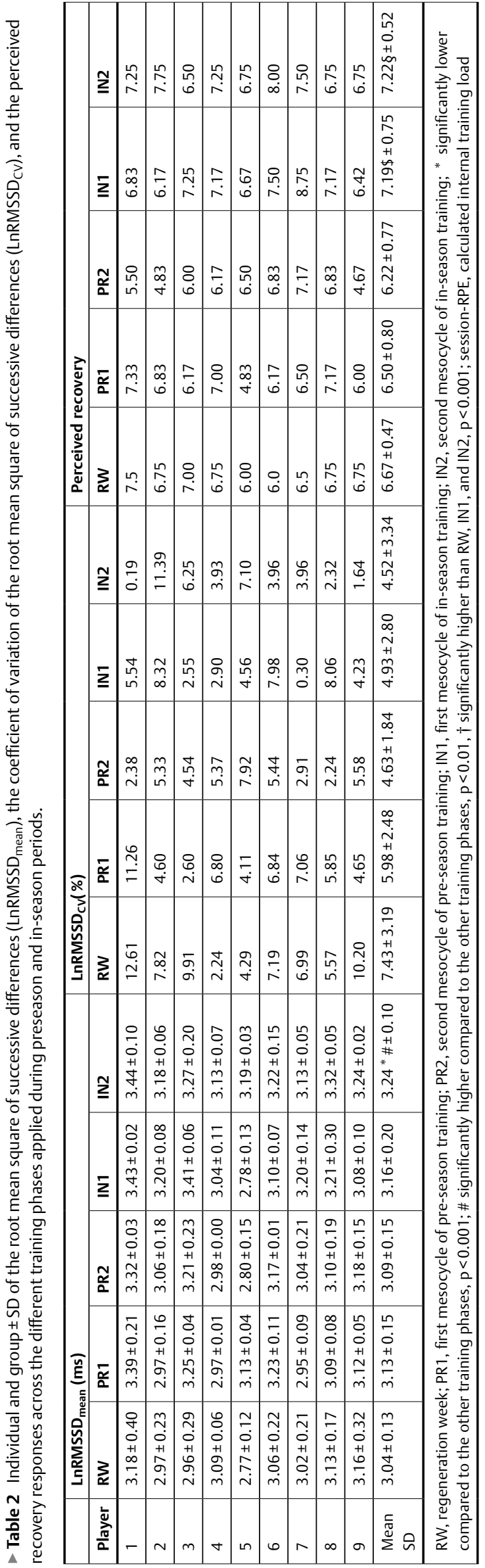

A major and unexpected finding of the present study was that despite the abrupt increment of training load during preseason training, $\operatorname{LnRMSSD}_{\text {mean }}$, $\operatorname{LnRMSSD}_{\mathrm{CV}}$, and perceived recovery remained unaltered compared to the regeneration week values. It has been shown that a considerable increment of internal training load may lead to a blunted cardiac parasympathetic response $[5,19,20]$. For instance, lellamo et al. [20] showed that endurance athletes who increased their training load up to $75-100 \%$ of maximum presented an increased resting heart rate and low-frequency components of R-R interval and decreased high-frequency components of R-R interval. Furthermore, Figueiredo et al. [5] reported that significant reductions in LnRMSSD may occur when training load increases more than $100 \%$ compared to baseline training. In this regard, recent findings in water polo [19] reported that a large increase $(442 \%)$ in internal training load resulted in reduced heart rate recovery response after a standardized swim test. In the present study, however, the training load applied in the preseason was higher by $60-70 \%$ compared to the regeneration week. Further, our participants were elite-level players with long training and playing experience. As such, we postulate that the present variations in training load can be considered relatively small to induce a meaningful cardiac autonomic perturbation. It is also likely that a twoweek period of increased training load is not long enough to induce detectable physiological alterations despite a tendency for a decreased LnRMSSD $D_{\text {mean }}$ during PR2. In light of this, the unchanged group values of $\mathrm{LnRMSSD}_{\text {mean }}$ and $\mathrm{LnRMSSD}_{\mathrm{cv}}$ indicate that during the preseason training period elite water-polo players are able to tolerate up to $\sim 12000$ au $\cdot$ week $^{-1}$ without presenting significant cardiac autonomic perturbations.

Moreover, previous studies have shown that the training content may affect the participants' sympathetic/parasympathetic balance $[21,22]$. Considering the players' training status as well as the training program, it appears that the overall training load applied in the preseason was not stressful enough to reduce players' vagal activity. Nonetheless, the current findings should be interpreted with caution because group data may mask the inter-individual variability in HRV. Actually, we found that some of our players showed negative responses to preseason training demonstrating decrements in Ln$\mathrm{RMSSD}_{\text {mean }}(\triangleright$ Table 2$)$. The reduced LnRMSSD mean along with the concominant increments in LnRMSSDcv and decrements in perceived recovery scores, which were observed in some of the players in the preseason, have been previously interpreted as overreaching signs [10]. Hence, along with group data, it might be of importance to consider the individual responses to training as well [10].

Furthermore, we demonstrated that the reduction of training load by $30 \%$ during in-season compared to preseason training was accompanied by a meaningful increase of LnRMSSD $_{\text {mean }}$. Indeed, LnRMSSD $_{\text {mean }}$ increased in IN2 by $7 \%$ and $5 \%$ compared to the regeneration week and PR2 respectively, indicating that the reduced training loads induced a shift towards parasympathetic predominance. In alignment with the present findings, recent studies in soccer and water polo players observed that the reduction of training and match load coincided with increased LnRMSSD [4,5]. It is noteworthy that despite the large variation in individual responses observed in the preseason, almost all players showed positive responses to training and competition in-season. Most probably, the significant increase of LnRMSSD $_{\text {mean }}$ together with the improve- 
- Table 3 Individual and group correlations between internal training load and the root mean square of successive differences (LnRMSSD) during preseason and in-season training periods.

\begin{tabular}{|l|c|c|c|c|}
\hline Player & Preseason training & p-value & In-season training & p-value \\
\hline 1 & -0.74 & 0.01 & 0.11 & 0.76 \\
\hline 2 & -0.03 & 0.90 & 0.33 & 0.32 \\
\hline 3 & -0.25 & 0.47 & 0.09 & 0.79 \\
\hline 4 & -0.61 & 0.06 & -0.02 & 0.94 \\
\hline 5 & -0.32 & 0.37 & -0.11 & 0.74 \\
\hline 6 & 0.05 & 0.88 & -0.02 & 0.96 \\
\hline 7 & 0.23 & 0.52 & -0.06 & 0.86 \\
\hline 8 & -0.03 & 0.93 & 0.00 & 0.98 \\
\hline 9 & -0.27 & 0.45 & -0.43 & 0.19 \\
\hline RM r & -0.26 & 0.02 & -0.02 & 0.88 \\
\hline RM r, repeated measures correlation & & & \\
\hline
\end{tabular}

ment of perceived recovery observed in-season suggests supercompensation and an augmented readiness to play. The present findings are in alignment with previous observations in elite water polo players, where following an overload period of training a meaningful reduction of internal training load was accompanied by significant increments of LnRMSSD $_{\text {mean }}$ [4] along with improvements in swimming performance and wellness scores [23]. Furthermore, the non-significant changes in LnRMSSD ${ }_{\mathrm{Cv}}$ values during inseason training reduced compared to preseason training probably imply that players coped well with the training load and adapted to the training stimuli [8]. Moreover, the lack of significant variation in $\mathrm{LnRMSSD}_{\mathrm{CV}}$ along with the improved perceived recovery during the in-season training likely suggest lower autonomic stress induced by training or facilitated recovery after training [24]. In this regard, previous findings from high-level water polo players indicated that a similar training manipulation (reduction of training load up to $30 \%$ compared to overload training) resulted in a significant increase in swimming performance and improved the wellness status of participants [23].

In accordance with previous reports $[4,8]$ showing unclear relationships between internal training load and HRV, we showed that despite the meaningful relationship between ITL and LnRMSSD mean in the preseason, a weak association was apparent thereafter (i.e., in-season). This was probably due to the fact that the session-RPE method is likely adequate to quantify the total training load applied in the preseason, in which players usually do not participate in official games. Conversely, the lack of association between the session RPE and LnRMSSD mean might be related to the presence of nontraining stressors (i.e., travel and days off) but inherent to the context of the competition schedule of an elite-level team.

Despite their importance, the present findings are limited by the relatively small sample size of elite players. Future studies with a larger group of elite players are required to investigate cardiac autonomic responses or adaptations elicited after different phases of training. Moreover, we acknowledge the absence of a swim test during the various training phases to connect cardiac responses with performance. However, in a real training scenario, the application of pre-post tests is difficult to implement in-season owing to time constraints imposed by the congested calendar including training sessions and consecutive national and international matches. Nonetheless, an improvement in swimming performance could be expected as a result of such training manipulation [23]. Additionally, we should consider the absence of inflammatory, muscle damage as well as neuro-endocrine measures as limitations. As it has been also recommended [10] that such measures be included in future studies in order to determine the relationship between recovery status of the players with HRV variables.

\section{Conclusion}

The current study suggests that cardiac autonomic perturbations during preseason training do not occur when an increment of internal training load is lower than $60-70 \%$. Nonetheless, the reduction of training load by $30 \%$ in-season was accompanied by significant increases in LnRMSSD $D_{\text {mean }}$ together with improvements in perceived recovery status. This implies that the training periodization presently applied was successful and probably led elite players in supercompensation associated with increased parasympathetic activity.

\section{Acknowledgements}

We would like to thank the players for their participation in this study and the coach of the team for his cooperation.

\section{Conflict of interest}

The authors declare that they have no conflict of interest.

\section{References}

[1] Botonis PG, Toubekis AG, Platanou TI. Physiological and tactical on-court demands of water polo. J Strength Cond Res 2019; 33 : 3188-3199

[2] Tessitorre A, Perroni F, Meeusen R et al. Coordination of soccer players during preseason training. J Strength Cond Res 2011; 25: 3059-3069 
[3] Stanley J, Peake JM, Buchheit M. Cardiac parasympathetic reactivation following exercise: implications for training prescription. Sports Med 2013; 43: 1259-1277

[4] Botonis PG, Smilios I, Platanou TI et al. Effects of an international tournament on heart rate variability and perceived recovery in elite water polo players. J Strength Cond Res 2020, doi:10.1519/ JSC.0000000000003783

[5] Figueiredo DH, Figueiredo DH, Moreira A et al. Effect of overload and tapering on individual heart rate variability, stress tolerance, and intermittent running performance in soccer players during preseason. J Strength Cond Res 2019; 33: 1222-1231

[6] Flatt AA, Esco MR. Smartphone-derived heart rate variability and training load in women's soccer team. Int J Sports Physiol Perform 2015; 10: 994-1000

[7] Flatt AA, Esco MR. Evaluating individual training adaptation with smartphone-derived heart rate variability in a collegiate female soccer team. J Strength Cond Res 2016; 30: 378-385

[8] Nakamura FY, Pereira LA, Rabelo FN et al. Monitoring weekly heart rate variability in futsal players during the preseason: The importance of maintaining high vagal activity. J Sports Sci 2016; 34: 2262-2268

[9] Flatt AA, Esco MR, Nakamura FY. Individual heart rate variability responses to preseason training in high level female soccer players. J Strength Cond Res 2017; 31: 531-538

[10] Flatt AA, Esco MR, Nakamura FY et al. Interpreting daily heart rate variability changes in collegiate female soccer players. J Sports Med Phys Fitness 2017; 57: 907-915

[11] Boullosa DA, Abreu L, Nakamura FY et al. Cardiac autonomic adaptations in elite Spanish soccer players during preaseason. Int J Sports Physiol Perform 2013; 8: 400-409

[12] Flatt AA, Howells D. Effects of varying training load on heart rate variability and running performance among an Olympic rugby sevens team. J Sci Med Sports 2019; 22: 222-226

[13] Harriss DJ, MacSween A, Atkinson G. Ethical standards in sport and exercise science research: 2020 update. Int J Sports Med 2019; 40: 813-817
[14] Paul D], Tomazoli G, Nassis GP. Match-related time course of perceived recovery in youth football players. Int J Sports Physiol Perform 2019; 14: 339-342

[15] Foster C, Florhaug JA, Franklin J et al. A new approach to monitoring exercise training. J Strength Cond Res 2001; 15: 109-115

[16] Lupo C, Capranica L, Tessitore A. The validity of session-RPE method for quantifying training load in water polo. Int J Sports Physiol Perform 2014; 9: 656-660

[17] Laurent CM, Green GM, Bishop PA. A practical approach to monitoring recovery: development of a perceived recovery status scale. J Strength Cond Res 2001; 25: 620-628

[18] Bland JM, Altman DG. Calculating correlation coefficient with repeated observations: Part 1-Correlation within subjects. BMJ 1995; 310: 446

[19] Botonis PG, Arsoniadis GG, Platanou TI et al. Heart rate recovery responses after acute training load changes in top-class water polo players. Eur J Sport Sci 2020, doi:10.1080/17461391.2020.1736181

[20] lellamo F, Legramante JM, Pigozzi F et al. Conversion from vagal to sympathetic predominance with strenuous training in high-performance world class athletes. Circulation 2002; 105: 2719-2724

[21] Buchheit M, Laursen PB, Ahmaidi S. Parasympathetic reactivation after repeated sprint exercise. Am J Physiol Heart Circ Physiol 2007; 293: $\mathrm{H} 133-\mathrm{H} 141$

[22] Costa JA, Brito J, Nakamura FY et al. Effects of late-night training on "slow-wave sleep episode" and hour-by-hour derived nocturnal cardiac autonomic activity in female soccer players. Int J Sports Physiol Perform 2018; 13: 638-644

[23] Botonis PG, Toubekis AG, Platanou TI. Training loads, wellness and performance before and during tapering for a water polo tournament. J Hum Kinet 2019; 27: 131-141

[24] Seiler S, Haugen O, Kuffel E. Autonomic recovery after exercise in trained athletes: intensity and duration effects. Med Sci Sports Exerc 2007; 39: 1366-1373 